\title{
All-optical switching based on controlled energy transfer between nanoparticles in film arrays
}

\author{
David S. Bradshaw and David L. Andrews ${ }^{\mathrm{a}}$ \\ ${ }^{a}$ Nanostructures and Photomolecular Systems, School of Chemical Sciences, \\ University of East Anglia, Norwich NR4 7TJ, United Kingdom \\ david.andrews@physics.org
}

\begin{abstract}
The potentiality to exert optical control, over the migration of electronic excitation energy between particles with suitably disposed electronic levels, affords a basis for all-optical switching. Implemented in a configuration with nanoparticles arrayed in thin films, the process can offer an ultrafast parallel-processing capability. The mechanism is a nearfield transfer of energy from donor nanoparticles in one layer (written into an electronically excited state by the absorption of light) to counterpart acceptors; the transfer effect proves amenable to activation by non-resonant laser radiation. The possibility of optical control arises under conditions where the donor-acceptor energy transfer is rigorously forbidden in the absence of laser light, either on the grounds of symmetry or energetics. Under such conditions, optical switching can be produced by the throughput of a single off-resonant beam or, with more control options, by two coincident beams. In model electrodynamical calculations the transfer fidelity, signifying the accuracy of mapping an input to its designated output, can be identified and cast in terms of key optical and geometric characteristics. The results show that, at reasonable levels of laser intensity, cross-talk drops to insignificant levels. Potential applications extend beyond simple switching into all-optical elements for logic gates and optical buffers.
\end{abstract}

Keywords: optical switching, thin films, nanoparticles, energy transfer, nonlinear optics.

\section{INTRODUCTION}

Although it is famously considered unfeasible for photons to directly interact with each other [1], there is a variety of means whereby, through an engagement of interactions with matter, light can itself be subject to optical control. Implemented in a form of an all-optical switch, any such process can offer immediate advantages over most electronic counterparts - these advantages generally centering on an ultrafast speed of operation and greatly diminished losses. As such, photonic interactions in which light is controlled by light have a credible potential to entirely revolutionize much of the computing and telecommunications industry. Numerous technical strategies have been entertained, and many are the subject of vigorous ongoing research. These include: semiconductor quantum wells systems [2-5], where optical switching is conceived as an exploitation of optical saturation - although an alternative method, based on atomic quantum interference in electromagnetic induced transparency has recently been demonstrated [6-8]; photonic crystals with cross-waveguide geometries, offering switching action through the optical Kerr effect [9-11]; surface plasmon polariton media, in which light-induced dielectric modification at an interface affects the transmission of throughput radiation [12-14]; and various schemes based on films of bacteriorhodopsin, a light harvesting protein with unique non-linear optical properties [15-17]. One possibility that has only recently begun to attract attention is an all-optical switching mechanism based on the optical control of resonance energy transfer (RET) between particles.

When RET occurs spontaneously, following the absorption of light, it usually represents the principal process for the intermolecular translation of electronic energy, from the sites of 
initial optical excitation [18]. However, under suitable conditions such processes of energy transfer can be activated or deactivated by (non-resonant) optical stimulation. In many features, optical switches based on this principle differ strikingly from the majority of previously proposed schemes. Here, photonic switching is localized in particle pairs rather than ensembles, and the mechanism allows much shorter operating wavelengths to be engaged, i.e. the ultraviolet or visible range, in contrast to the infrared. Spontaneous RET is a well-studied phenomenon that involves relocation of energy from an electronically excited donor $A$, to an acceptor $B$ in its ground state. By input of an auxiliary laser field, such energy transfer may be enhanced by a nonlinear response mechanism termed laser-assisted resonance energy transfer (LARET), whose efficiency scales linearly with the laser intensity $[19,20]$. Optically controlled resonance energy transfer (OCRET) - the mechanistic basis for the study reported here and illustrated by Fig. 1 - is a related process, differing from LARET in that spontaneous RET is completely excluded. In previous work [21] this preclusion was founded upon a geometric configuration. While feasible, this may be difficult in practice; alternative methods to exclude spontaneous RET are based on symmetry and energetic grounds (vide infra). By application of the off-resonant laser beam the transfer of energy is activated, effecting all-optical switching action.

The following analysis begins by developing the fundamental electrodynamic theory for the OCRET mechanism, with consideration of a donor-acceptor pair in a single- or dual-beam configuration (Section 2). The investigation is then extended to an arrangement that could be useful for realistic all-optical switches, taking into account the requirement for a multiplicity of such donor-acceptor partners to operate independently, without significant cross-talk. This arrangement is envisaged to involve nanoparticles (representing pixels of the array) written into thin-film deposits on a pair of parallel substrates. The specific geometric configuration to be examined is a square-packed configuration (Section 3). Further performance issues are addressed in the concluding Section 4.

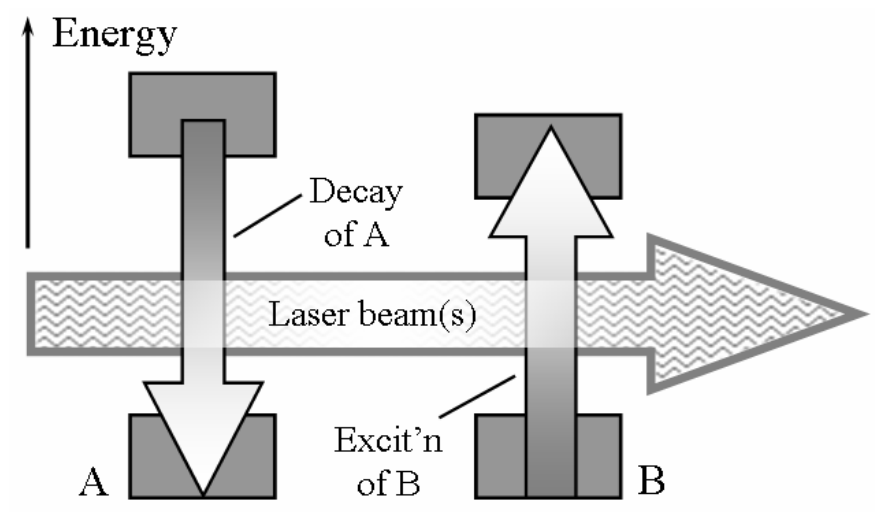

Fig. 1. General schematic of the OCRET mechanism.

\section{MECHANISM FOR ALL-OPTICAL SWITCHING}

The OCRET mechanism entails the simultaneous coupling of RET with pairwise absorption and stimulated re-emission of the incident laser. As depicted in Fig. 2, this involves photon absorption and emission at the donor and acceptor, respectively, or vice-versa; both result in excitation transfer from $A$ to $B$. Each particle involves two photon interactions and, thus, the electronic transitions must be two-photon allowed within both $A$ and $B$. To exclude spontaneous RET on symmetry grounds, one of these transition must be one-photon forbidden. This is now to be discussed for the single-beam configuration. 


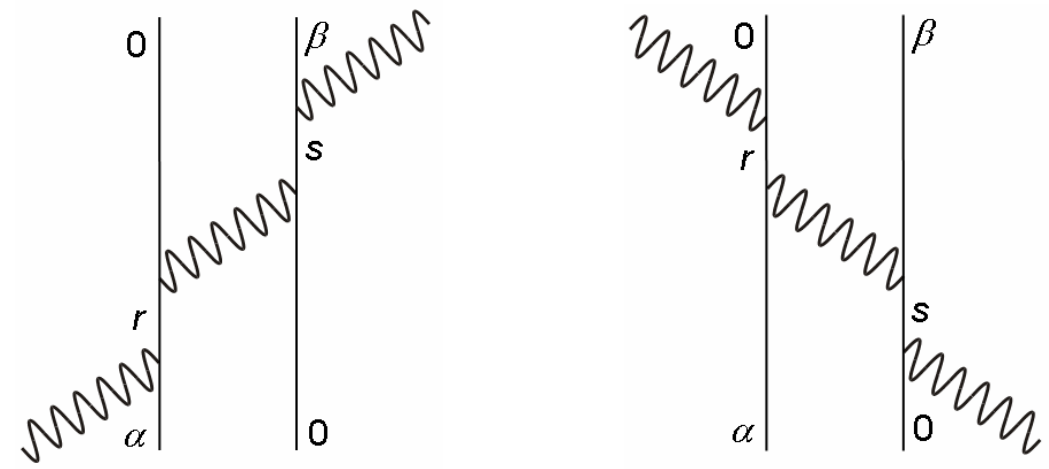

Fig. 2. Feynman diagrams for OCRET. Here, $|0\rangle$ represents a particle in the ground state; $|\alpha\rangle$ and $|\beta\rangle$ relate to the excited state of the donor (on the left) and acceptor (right), respectively, with $|r\rangle$ and $|s\rangle$ as the corresponding intermediates.

\subsection{Single-beam configuration}

To specifically determine the transfer efficiency for the OCRET mechanism with a single input auxiliary beam, a time-dependent perturbation theory method is needed. As is required for optical switching action based on selection rules, the electronic transitions within donor $A$ or acceptor $B$ are one-photon forbidden. Therefore, no spontaneous RET is present and as a result the excitation transfer occurs only through a mechanism that engages the auxiliary input beam. In the near-field, the corresponding quantum amplitude involving two-photon transitions is given by [21];

$$
M_{f i}^{(4)}=\left(\frac{m \hbar c k}{8 \pi \varepsilon_{0}^{2} V R^{3}}\right) e_{i} \bar{e}_{l}\left(\delta_{j k}-3 \hat{R}_{j} \hat{R}_{k}\right)\left(S_{i j}^{A}(k) S_{l k}^{B}(-k)+S_{i j}^{B}(k) S_{l k}^{A}(-k)\right),
$$

where $m$ is the number of photons (proportional to intensity) in the quantization volume $V$, and the implied summation convention for repeated Cartesian tensor indices is employed. Furthermore, e and $\hbar c k$ denote the polarization vector (an overbar denoting complex conjugation) and energy of the input photon, respectively. Each of the two terms of Eq. (1), illustrated by the Feynman diagrams of Fig. 2, represents a fourth-order photonic interaction event, necessitating deployment of a fourth-order perturbation treatment as indicated by the superscript of $M_{f i}$. Also in (1) is the generalized polarizability, $S_{i j}^{\xi}( \pm k)$, explicitly given by;

$$
S_{i j}^{\xi}( \pm k)=\sum_{r}\left\{\frac{\mu_{i}^{f r} \mu_{j}^{r i}}{E_{r f} \pm \hbar c k}+\frac{\mu_{j}^{f r} \mu_{i}^{r i}}{E_{r i} \mp \hbar c k}\right\} .
$$

Here the transition dipole moments are designated by the shorthand notation $\boldsymbol{\mu}^{x y}=\langle x|\boldsymbol{\mu}| y\rangle$, energy differences being expressed in the form $E_{x y}=E_{x}-E_{y}$. Further, $f$ signifies the final electronic state of particle $\xi, i$ is the initial state, and $r, s$ are intermediates. To a good approximation, let it be assumed that the sums of Eq. (2) are limited to the three states that determine the most prominent optical features. These are denoted $|0\rangle,|\sigma\rangle,|\alpha\rangle$ for donor $A$, and $|0\rangle,|\tau\rangle,|\beta\rangle$ for acceptor $B$ - where $|\alpha\rangle$ and $|\beta\rangle$ are the levels between which energy transfer occurs. It is also expedient to select a frequency for the input radiation that has a 
resonance offset with respect to the positioning of these levels, a condition expressible as $E_{\sigma \alpha}=\hbar c k+\Delta E_{A}$, where $\Delta E_{A}$ is a non-zero energy with magnitude significantly lower than a typical transition energy. An expression of similar form, $E_{\tau \beta}=\hbar c k+\Delta E_{B}$, is assumed for $B$. The outcome of applying these conditions is that one summand is significantly larger in magnitude than the rest. Hence, Eq. (1) becomes:

$$
M_{f i}^{(4)}=\left(\frac{m \hbar c k\left(\mathbf{e} \cdot \boldsymbol{\mu}^{\sigma \alpha}\right)\left(\overline{\mathbf{e}} \cdot \boldsymbol{\mu}^{\beta \tau}\right)}{8 \pi \varepsilon_{0}^{2} V R^{3} \Delta E_{A} \Delta E_{B}}\right)\left(\boldsymbol{\mu}^{0 \sigma} \cdot \boldsymbol{\mu}^{\tau 0}-3\left(\boldsymbol{\mu}^{0 \sigma} \cdot \hat{\mathbf{R}}\right)\left(\boldsymbol{\mu}^{\tau 0} \cdot \hat{\mathbf{R}}\right)\right) .
$$

Next, we introduce a Cartesian basis in which the donor-acceptor displacement vector $\mathbf{R}$ is identified with the $z$-direction. The vectors $\hat{\boldsymbol{\mu}}_{A} \equiv \hat{\boldsymbol{\mu}}^{0 \alpha}$ and $\hat{\boldsymbol{\mu}}_{B} \equiv \hat{\boldsymbol{\mu}}^{\beta 0}$ are chosen unambiguously as being directed in the $\hat{\mathbf{i}}$ and $\hat{\mathbf{j}}$ directions respectively and, with a judicious choice that exploits symmetry grounds, $\hat{\boldsymbol{\mu}}^{0 \sigma}=\hat{\mathbf{k}}$ and $\hat{\boldsymbol{\mu}}^{\sigma \alpha}=\hat{\mathbf{j}}$. Also, it can be arranged that $\hat{\boldsymbol{\mu}}^{\tau 0}=\hat{\mathbf{k}}$ and $\hat{\boldsymbol{\mu}}^{\beta \tau}=-\hat{\mathbf{i}}$. For convenience $A$ and $B$ are chosen to belong to identical symmetry groups, although the same calculational method allows for systems where this is not the case. Therefore, employing this configuration, Eq. (3) is rewritten as;

$$
M_{f i}^{(4)}=\left(\frac{-m \hbar c k|\mu|^{4} \kappa^{\prime}}{8 \pi \varepsilon_{0}^{2} V r^{3} \Delta E_{A} \Delta E_{B}}\right) \sin ^{2} \phi \cos \theta \sin \theta,
$$

where the orientation factor is $\kappa^{\prime}=\left(R^{2}-3 r^{2}\right) / R^{2}=-2$, given that $\mathbf{R}=r \hat{\mathbf{k}}, r$ being the displacement of $B$ from $A$. Furthermore, the angles $\phi$ and $\theta$ denote the orientations of $\mathbf{e}$ with respect to $\hat{\mathbf{k}}$ and $\hat{\mathbf{i}}$, respectively (with the input photon polarization assumed linear). In addition, all transition dipole moments will have broadly similar magnitude - thus, for simplicity, these are assumed to be equal. Equation (4) clearly delivers a non-zero result, unlike the precluded Förster transfer. The time-dependent probability, $P(t)$, that the energy transfer process can proceed is secured from the Fermi's Golden Rule, here expressible in the following form where $\rho_{f}$ is the density of states associated with the donor and acceptor excited state spectral overlap;

$$
P(t)=\frac{2 \pi \rho_{f}}{\hbar} \int_{0}^{t}\left|M_{f i}^{(2)}+M_{f i}^{(4)}+\ldots\right|^{2} \mathrm{~d} t
$$

with higher-order quantum amplitudes rapidly diminishing in magnitude. In equation (5) the second-order contribution $M_{f i}^{(2)}$ corresponds to RET - a null quantity for the configurations described in this paper. Substituting equation (4) into (5) gives the expression;

$$
P(t)=\frac{4 K C^{2} J^{\prime}}{r^{6}} \sin ^{4} \phi \cos ^{2} \theta \sin ^{2} \theta
$$

parametrized in terms of molecular properties $K=|\mu|^{4} \rho_{f} / 8 \pi \varepsilon_{0}^{2} \hbar$ and $C=|\mu|^{2} / 2 c \varepsilon_{0} \Delta E_{A} \Delta E_{B}$ and the optical pulse property $J^{\prime}=\int_{0}^{t} I^{2}(t) \mathrm{d} t$, in which $I(t) \equiv m \hbar c^{2} k / V$ is the irradiance. It is noteworthy that with $\phi=0^{\circ}, \theta=0^{\circ}$ or $\theta=90^{\circ}$, no energy transfer to the acceptor occurs. Although not shown here there is a further mechanism that, as a result of the condition that $A$ and $B$ have identical symmetry, involves three-photon transitions at one particle 
(corresponding to three dipole transition events) and a one-photon transition at the other - see Fig. 3. This latter mechanism may well be active if only one of the particles is one-photon forbidden, but it proves much less efficient compared to the process of Fig. 2. The intricate analysis for this three-photon allowed mechanism is detailed within refs [21] and [22].
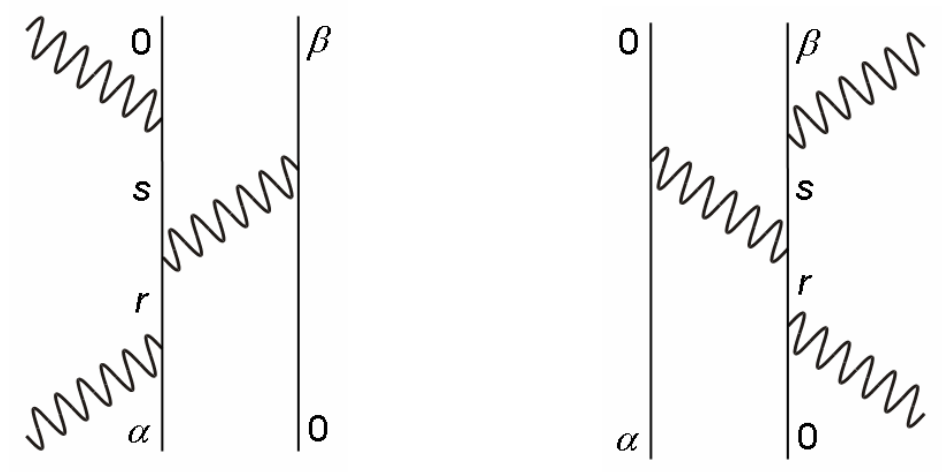

Fig. 3. Feynman diagrams for an optical control mechanism, involving three-photon interactions at one particle and one-photon interactions at the other. The symbols are identical to Fig. 2.

\subsection{Dual-beam configuration}

A dual-beam configuration is now considered. The earlier expressions may be recast to allow for the case where the $A-B$ system is simultaneously subject to the confluence of two beams of off-resonant laser radiation. By following the same procedure as above, $P(t)$ will be equivalent to Eq. (6) except for $J^{\prime}=\int_{0}^{t} I_{1}(t) I_{2}(t) \mathrm{d} t$, i.e. the intensities of both the incident and emission beams are engaged. Further, the values $\Delta E_{A}$ and $\Delta E_{B}$ are more easily manipulated. An obvious advantage of dual auxiliary beams over a single-beam set-up is the greater number of independently controllable experimental parameters, resulting in enhanced control of transfer efficiency between the $A-B$ pair [23]. A further benefit is the capacity to produce optically switched energy transfer in systems with low spectral overlap. Specifically, the frequency difference between the input beams can be tuned to compensate for any mismatch between the donor and acceptor excited state energies (see Fig. 4).

\section{THIN-FILM ARRANGEMENT}

We now develop the single-beam donor-acceptor model of the last section into that of a potential all-optical switch. A proposed structure is based on the deposition of thin films on the juxtaposed surfaces of a pair of parallel substrates, where each film contains an ordered set of nanoparticles (henceforth termed pixels, to denote the anticipated implementation); one film comprises donor pixels, and the other, acceptors. We investigate a system where the pixels within the adjacent thin-films have a square-packed configuration with lattice constant $l$; within each film the pixels are equally spaced and identical (Fig. 5). It is assumed that each array lies on an $(x, y)$ plane. The configuration is constructed such that each constituent pixel within the donor film directly corresponds to a counterpart within the acceptor film; these pairs are coded by the coordinates $u$ and $v$ (integer values) and are displaced, one from each other, by $r \hat{\mathbf{k}}$. Furthermore, all nanoparticles in the donor and acceptor films are chosen to be oriented in the $\hat{\mathbf{i}}$ and $\hat{\mathbf{j}}$ directions, respectively. 


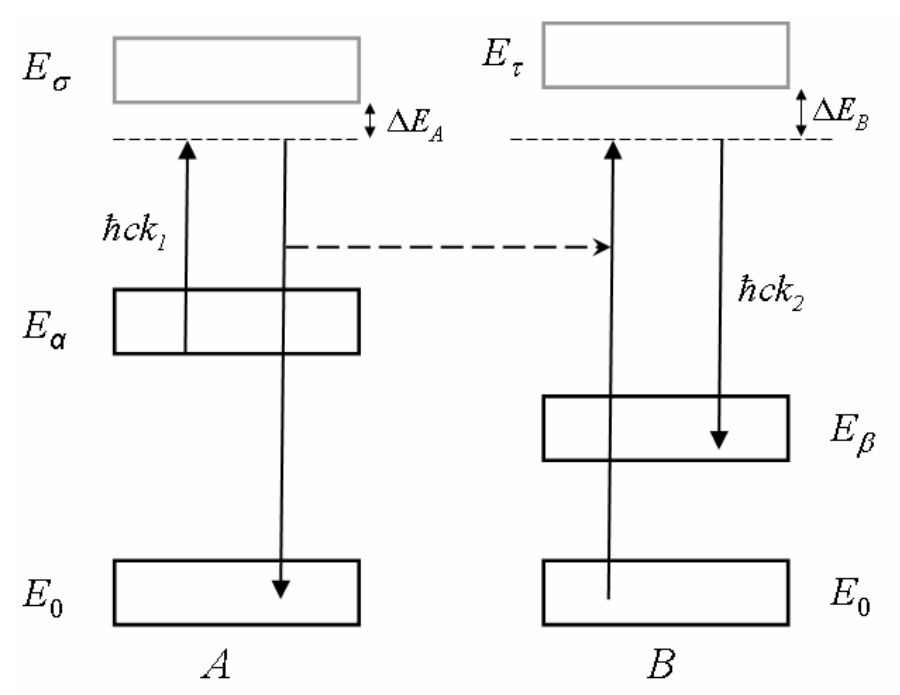

Fig. 4. Energetics scheme for OCRET from $A$ to $B$. Solid-head arrows denote the four transitions coupling the donor decay $|\alpha\rangle \rightarrow|0\rangle$ and acceptor excitation $|0\rangle \rightarrow|\beta\rangle$, laser interactions denoted by the photon energies. Dotted lines denote virtual states, the closest real states $E_{\sigma}$ and $E_{\tau}$ being offset in energy by $\Delta E_{A}$ and

$\Delta E_{B}$, respectively. The dashed arrow signifies energy transfer.

It is expedient to focus on processes by means of which energy can transfer from any one specific excited donor (for convenience located at $u=0, v=0$ ) to any other pixel. First, consider energy relocation to an arbitrary pixel within the acceptor film. To find an expression for $P(t)$, noting spontaneous RET is again excluded, the following is determined;

$$
M^{(4)}=\left(\frac{-m \hbar c k|\mu|^{4}}{8 \pi \varepsilon_{0}^{2} l^{3} V \Delta E_{A} \Delta E_{B}}\right)\left(\frac{u^{2}+v^{2}-2 r^{\prime 2}}{\left(u^{2}+v^{2}+r^{\prime 2}\right)^{\frac{5}{2}}}\right) \sin ^{2} \phi \cos \theta \sin \theta .
$$

Employing Eqs (7) with (5), we have;

$$
P(t)=\frac{K C^{2} J^{\prime}\left(u^{2}+v^{2}-2 r^{\prime 2}\right)^{2}}{l^{6}\left(u^{2}+v^{2}+r^{\prime 2}\right)^{5}} \sin ^{4} \phi \cos ^{2} \theta \sin ^{2} \theta,
$$

where $J^{\prime}=\int_{0}^{t} I^{2}(t) \mathrm{d} t, I$ being the irradiance of the input laser beam.

Having secured this result, the context makes it also necessary to consider cases of energy transfer from one initially excited donor pixel to another within the same (donor) film. The resulting expression is analogous to Eq. (8), but with $r^{\prime}$ taken as zero. In the following, we also take the same value of $K$ for such intra-film transfer, recognizing it as marking an upper limit on the extent to which in-film transfer might compromise the sought transfer to the acceptor film. In practice the value will likely be significantly lower. The reason is that the initial excitation of any single donor will usually be followed by rapid intramolecular decay to 


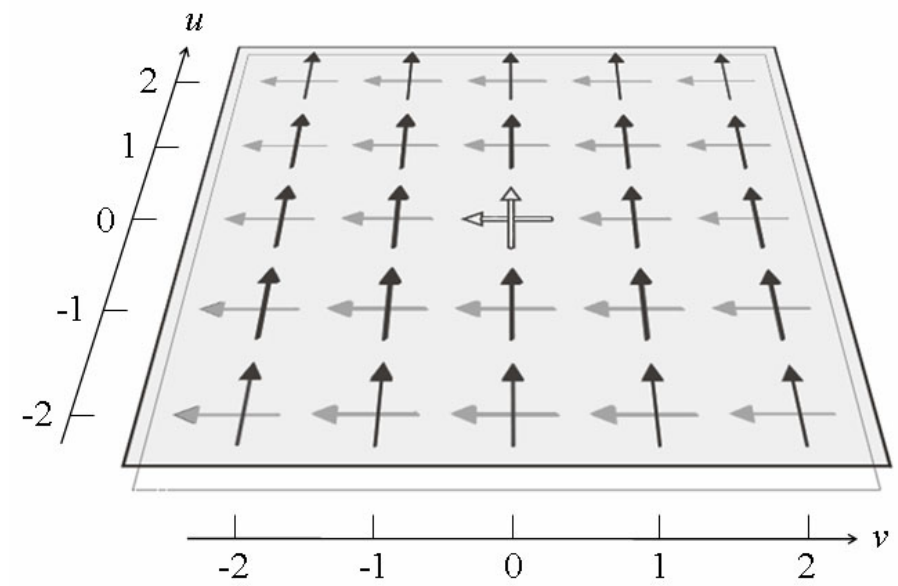

Fig. 5. Structure of the square-packed configuration (viewed from above). Both substrates lie in the $(x, y)$-plane, with all donor transition moments (black) in the upper film parallel to the $x$-axis, and all acceptor transition moments (gray) in the lower film parallel to the $y$-axis. Open arrows represent an arbitrary excited donor and its counterpart acceptor.

the energetically lowest part of the excited state continuum, before energy transfer ensues. Consequently the density of states for any donor film pixel acting as acceptor of the energy exchange will be small. By contrast, the usual spectroscopic gradient [24] that operates between the (necessarily chemically different) donor and acceptor particles will generally ensure energy delivery into a high-density part of the excited state continuum.

To quantify the cross-film transfer we now compare the efficiency of direct energy relocation, from an excited donor to its designated partner in the acceptor film (the sought destination, $S$ ), to the summed efficiencies for transfer to any other pixels within either array (cross-talk destinations, $C$ ). The dependence of the result on the aspect ratio is determined and graphically depicted as plots of $P(t)$ for different transfer destinations, as shown in Fig. 6 . Performing the calculations for the graph, typical parameter values are employed, namely: $\Delta E=3 \times 10^{-20} \mathrm{~J},|\mu|=1 \times 10^{-29} \mathrm{C} \mathrm{m}, \phi=90^{\circ}$ and $\theta=45^{\circ}$ (the optimum angles). It transpires that the probability of transfer between well-separated donors and acceptors (above $|u|,|v|=10)$ proves negligible. It is notable that the values ascribed to the variables affect the absolute transfer efficiency, but not the destination of the excitation. The fidelity, $F=S /(S+C)$, denotes the fraction of the transfer that delivers energy to the counterpart acceptor; this is plotted on a logarithmic scale and shown in Fig. 6 (inset). The laser input, assumed to be time-independent, is given a moderate value of $1 \times 10^{10} \mathrm{~W} \mathrm{~cm}^{-2}$. As the plots within Fig. 6 illustrate, the transfer of energy from the excited donor to the corresponding particle in the acceptor film greatly dominates all other transfer routes for an aspect ratio, $r^{\prime}$, up to $\sim 0.6$. Specifically, no less than $95 \%$ of the total excitation is transported to the required destination. As might be anticipated, this transfer fidelity is increasingly compromised when the aspect ratio is increased - see Fig. 6 (inset). It is interesting to note that the fidelity is independent of the laser intensity. The dual auxiliary beam configuration will give the same results and, thus, will not affect the destination of the excitation to any greater extent than the single-beam set-up. Therefore, no additional benefits are determined, in this instance, with the deployment of a two-beam configuration. The value of dual-beam implementation lies not in enhanced efficiency, but an increased number of experimentally controllable parameters. 


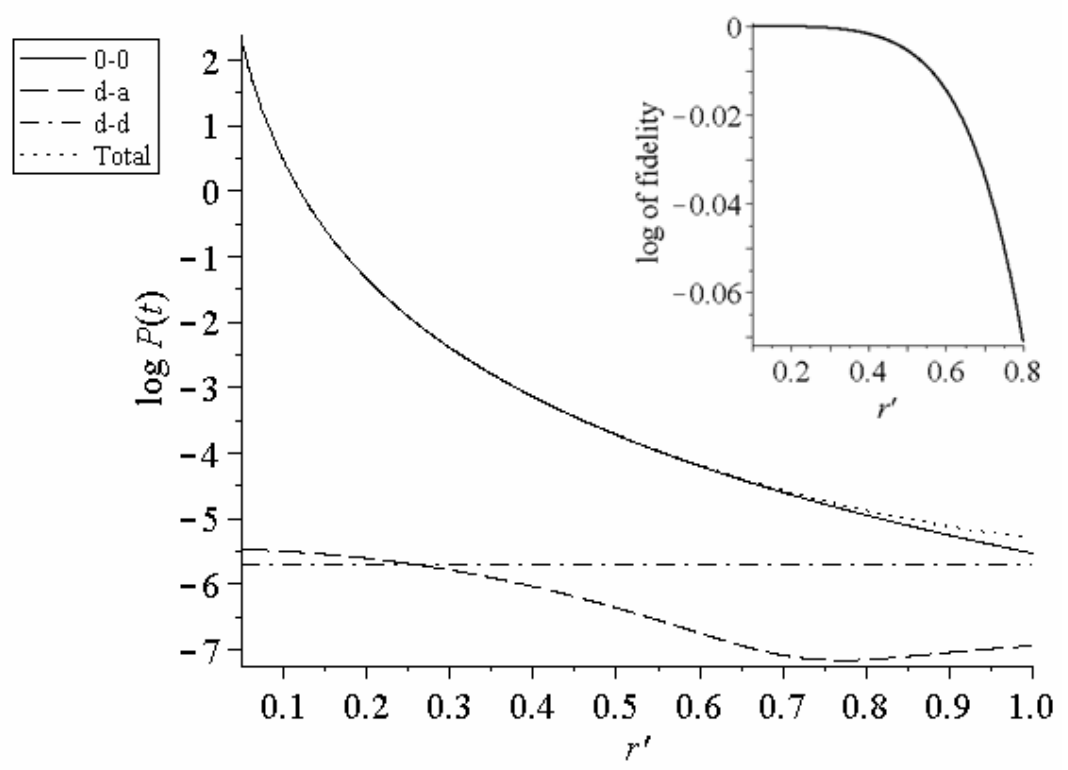

Fig. 6. Plot of $\log P(t)$, where $P(t)$ is the time-dependent probability, against the aspect ratio, $r^{\prime}$, for optical transfer from an excited nanoparticle in the donor square-lattice array to the required destination in the acceptor square array (0-0); also depicted are the 'cross-talk' probabilities for transfer to another particle in either the acceptor (d-a) or the donor (d-d) array, and the sum of all three transfer possibilities (Total). Inset: difference between logarithms of the $0-0$ and the sum probabilities for various $r^{\prime}$, signifying on a logarithmic scale the transfer fidelity; on the ordinate axis each -0.01 increment corresponds to $2.3 \%$ loss. Here, the intensity $I$ of the input laser is $1 \times 10^{10} \mathrm{~W} \mathrm{~cm}^{-2}$.

\section{DISCUSSION}

The foregoing analysis has addressed the necessary criteria and effective constraints surrounding the possible deployment of OCRET as a means of achieving all-optical switching. The proposed device architecture for such a switch involves thin-film deposits on parallel substrates - an ordered set of nanoparticles being contained within these films. The concept of transfer fidelity, signifying the accuracy of mapping input to designated output, has been introduced and its key determinants identified from the form of the calculated results. Single- and dual-beam configurations have been considered, the latter enabling conventional RET to be excluded solely on energetic grounds. For the envisaged switching device, both of the presented techniques to omit spontaneous RET are likely to prove more effective than the geometric condition employed in our earlier paper [21]. The detailed analysis has shown that it is possible, by judicious choice of the relative values of the film spacing and lattice constant, to achieve arbitrarily low amounts of cross-talk.

Generally, it may prove expedient to incorporate a suitably transparent ultra-thin spacer material as a separator between the donor and acceptor film layers. The results will be identical to those presented here, except that all quantum amplitudes will be multiplied by the appropriate Lorenz factor $\left(n^{2}+2\right) / 3$ - the symbol $n$ denoting the refractive index of the spacer material. Therefore, the absolute transfer efficiency will be enhanced by the inclusion of an ultra-thin spacer with suitable dielectric properties, although the directedness of the excitation delivery is unaltered by such a set-up. The deposition and tailoring of the nanoparticle components, in each active layer, is expected to be achieved by photolithography 
$[25,26]$. Through a novel form of this technique, the nanofabrication of structures similar to those envisaged have already been constructed $[27,28]$. Therefore, there is much promise that a workable device could be manufactured.

In terms of applications, the possible achievement of optical switching in an extensive parallel-processing unit introduces a number of potential applications, beyond simple switching. Logic gate construction - an example of which is given by ref. [29] - is an obvious possibility; the responsiveness to input modulation also suggests other forms of action, possibly leading to optical transistor configurations. Our results furthermore signify that, for example, pixel-based images, written by donor excitation, could be transferred with high fidelity to an acceptor film. In the realm of optical communications, possibilities might be built on the obvious capacity of such a system to act as an ultrafast information buffer; the high level of interest in such devices has already prompted others to explore 'slow-light' methods, where a host of more problematic limitations apply [30,31]. The systems we have presented offer numerous advantages: viable operation at short uv/visible wavelengths; the obviation of non-standard, expensive optical elements; the lack of any susceptibility to saturation problems; wide applicability to a host of molecular systems; a high information density optimized by using a single donor-acceptor pair for each bit of information; ultrafast response with high repetition rate, high efficiency, and nanoscale miniaturization.

\section{Acknowledgments}

We wish to thank the Leverhulme Trust for providing the financial support for this research.

\section{References}

[1] P. A. M. Dirac, The Principles of Quantum Mechanics, 4th ed., p. 9, Oxford University Press, London (1958).

[2] R. Akimoto, B. S. Li, K. Akita, and T. Hasama, "Ultrafast intersubband optical switching in II-VI-based quantum well for optical fiber communications," phys. stat. sol. (b) 243, 805-812 (2006) [doi:10.1002/pssb.200564715].

[3] Y. Li, A. Bhattacharyya, C. Thomidis, T. D. Moustakas, and R. Paiella, "Nonlinear optical waveguides based on near-infrared intersubband transitions in GaN/AIN quantum wells," Opt. Express 15, 5860-5865 (2007) [doi:10.1364/OE.15.005860].

[4] G. W. Cong, R. Akimoto, K. Akita, T. Hasama, and H. Ishikawa, "Low-saturationenergy-driven ultrafast all-optical switching operation in $(\mathrm{CdS} / \mathrm{ZnSe}) / \mathrm{BeTe}$ intersubband transition," Opt. Express 15, 12123-12130 (2007) [doi:10.1364/OE.15.012123].

[5] Y. Fedoryshyn, P. Strasser, P. Ma, F. Robin, and H. Jäckel, "Optical waveguide structure for an all-optical switch based on intersubband transitions in InGaAs/AlAsSb quantum wells," Opt. Lett. 32, 2680-2682 (2007) [doi:10.1364/OL.32.002680].

[6] P. Bermel, A. Rodriguez, S. G. Johnson, J. D. Joannopoulos, and M. Soljacic, "Singlephoton all-optical switching using waveguide-cavity quantum electrodynamics," Phys. Rev. A 74, 043818 (2006) [doi:10.1103/PhysRevA.74.043818].

[7] C. Y. Wang, Y. F. Chen, S. C. Lin, W. H. Lin, P. C. Kuan, and I. A. Yu, "Low-lightlevel all-optical switching," Opt. Lett. 31, 2350-2352 (2006) [doi:10.1364/OL.31.002350].

[8] J. P. Zhang, G. Hernandez, and Y. F. Zhu, "All-optical switching at ultralow light levels," Opt. Lett. 32, 1317-1319 (2007) [doi:10.1364/OL.32.001317].

[9] M. F. Yanik, S. H. Fan, M. Soljacic, and J. D. Joannopoulos, "All-optical transistor action with bistable switching in a photonic crystal cross-waveguide geometry," Opt. Lett. 28, 2506-2508 (2003) [doi:10.1364/OL.28.002506]. 
[10] N. Moll, R. Harbers, R. F. Mahrt, and G. L. Bona, "Integrated all-optical switch in a cross-waveguide geometry," Appl. Phys. Lett. 88, 171104 (2006) [doi:10.1063/1.2197931].

[11] M. R. Singh and R. H. Lipson, "Optical switching in nonlinear photonic crystals lightly doped with nanostructures," J. Phys. B 41, 015401 (2008)

[doi:10.1088/0953-4075/41/1/015401].

[12] C. Janke, J. G. Rivas, P. H. Bolivar, and H. Kurz, "All-optical switching of the transmission of electromagnetic radiation through subwavelength apertures," Opt. Lett. 30, 2357-2359 (2005) [doi:10.1364/OL.30.002357].

[13] G. A. Wurtz, R. Pollard, and A. V. Zayats, "Optical bistability in nonlinear surfaceplasmon polaritonic crystals," Phys. Rev. Lett. 97, 057402 (2006) [doi:10.1103/PhysRevLett.97.057402].

[14] D. E. Chang, A. S. Sorensen, E. A. Demler, and M. D. Lukin, "A single-photon transistor using nanoscale surface plasmons," Nature Physics 3, 807-812 (2007) [doi:10.1038/nphys708].

[15] Y. H. Huang, S. T. Wu, and Y. Y. Zhao, "All-optical switching characteristics in bacteriorhodopsin and its applications in integrated optics," Opt. Express 12, 895-906 (2004) [doi:10.1364/OPEX.12.000895].

[16] S. Roy, P. Sharma, A. K. Dharmadhikari, and D. Mathur, "All-optical switching with bacteriorhodopsin," Opt. Comm. 237, 251-256 (2004) [doi:10.1016/j.optcom.2004.04.013].

[17] J. Topolancik and F. Vollmer, "All-optical switching in the near infrared with bacteriorhodopsin-coated microcavities," Appl. Phys. Lett. 89, 184103 (2006) [doi:10.1063/1.2372711].

[18] D. L. Andrews and A. A. Demidov (Eds.), Resonance Energy Transfer, Wiley, Chichester, United Kingdom (1999).

[19] P. Allcock, R. D. Jenkins, and D. L. Andrews, "Laser assisted resonance energy transfer," Chem. Phys. Lett. 301, 228-234 (1999) [doi:10.1016/S0009-2614(98)01427-4].

[20] P. Allcock, R. D. Jenkins, and D. L. Andrews, "Laser-assisted resonance-energy transfer," Phys. Rev. A 61, 023812 (2000) [doi:10.1103/PhysRevA.61.023812].

[21] D. S. Bradshaw and D. L. Andrews, "Optically controlled resonance energy transfer: Mechanism and configuration for all-optical switching," J. Chem. Phys. 128, 144506 (2008) [doi:10.1063/1.2894319].

[22] D. L. Andrews, R. G. Crisp, and S. Li, "Single and dual beam optical switching of resonance energy transfer," J. Chem. Phys. 127, 174702 (2007) [doi:10.1063/1.2781392].

[23] D. L. Andrews, "Optically switched energy transfer: Twin-beam off-resonance control," Phys. Rev. Lett. 99, 023601 (2007) [doi:10.1103/PhysRevLett.99.023601].

[24] D. L. Andrews and J. Rodríguez, "Resonance energy transfer: Spectral overlap, efficiency, and direction," J. Chem. Phys. 127, 084509 (2007) [doi:10.1063/1.2759489].

[25] B. D. Gates, Q. Xu, M. Stewart, D. Ryan, C. G. Willson, and G. M. Whitesides, "New approaches to nanofabrication: Molding, printing, and other techniques," Chem. Rev. 105, 1171-1196 (2005) [doi:10.1021/cr030076o].

[26] R. Garcia, R. V. Martinez, and J. Martinez, "Nano-chemistry and scanning probe nanolithographies," Chem. Soc. Rev. 35, 29-38 (2006) [doi:10.1039/b501599p].

[27] W. Wu, D. Dey, O. G. Memis, A. Katsnelson, and H. Mohseni, "A novel self-aligned and maskless process for formation of highly uniform arrays of nanoholes and nanopillars," Nanoscale Res. Lett. 3, 23-127 (2008) [doi:10.1007/s11671-008-9124-6]. 
[28] H.-W. Huang, P. Bhadrachalam, V. Ray, and S. J. Koh, "Single-particle placement via self-limiting electrostatic gating," Appl. Phys. Lett. 93, 073110 (2008)

[doi:10.1063/1.2972042].

[29] E. K. L. Yeow and R. P. Steer, "Energy transfer involving higher electronic states: a new direction for molecular logic gates," Chem. Phys. Lett. 377, 391-398 (2003) [doi:10.1016/S0009-2614(03)01150-3].

[30] R. W. Boyd and P. Narum, "Slow- and fast-light: fundamental limitations," J. Mod. Opt. 54, 2403-2411 (2007) [doi:10.1080/09500340701639649].

[31] K. L. Tsakmakidis, A. D. Boardman, and O. Hess, "'Trapped rainbow' storage of light in metamaterials," Nature 450, 397-401 (2007) [doi:10.1038/nature06285]. 